\title{
SOME CHARACTERIZATIONS OF SEMI-BLOCH FUNCTIONS
}

\author{
RAUNO AULASKARI AND PETER LAPPAN
}

(Communicated by Clifford J. Earle)

\begin{abstract}
A function $f$ analytic in the unit disk is called a semi-Bloch function if, for each complex number $\lambda$, the function $g_{\lambda}(z)=\exp (\lambda f(z))$ is a normal function. We give both an analytic and a geometric characterization of semi-Bloch functions, together with some examples to show that semi-Bloch functions are not closed under either addition or multiplication.
\end{abstract}

\section{INTRODUCTION AND PRELIMINARIES}

A function $f$ analytic in the unit disk $D$ is called a Bloch function if

$$
\sup \left\{\left|f^{\prime}(z)\right|\left(1-|z|^{2}\right): z \in D\right\}=\|f\|_{B}<\infty .
$$

The collection of Bloch functions forms an interesting and much studied linear space (see, for example, $[2,3]$ ). A function $f$ meromorphic in $D$ is called a normal function if

$$
\sup \left\{f^{\#}(z)\left(1-|z|^{2}\right): z \in D\right\}=c_{f}<\infty,
$$

where

$$
f^{\#}(z)=\frac{\left|f^{\prime}(z)\right|}{1+|f(z)|^{2}}
$$

is the spherical derivative of $f$ (see [6]). If $f(z)$ is a Bloch function and $g(z)=\exp (f(z))$, then

$$
g^{\#}(z)\left(1-|z|^{2}\right)=\frac{|\exp (f(z))|}{1+|\exp (f(z))|^{2}}\left|f^{\prime}(z)\right|\left(1-|z|^{2}\right) \leq\|f\|_{B}
$$

and thus $g$ is a normal function. This fact was noted by Tse [7, Theorem 11, p. 70]. A function $f$ analytic in $D$ is called a semi-Bloch function if, for each complex number $\lambda$, the function $g_{\lambda}(z)=\exp (\lambda f(z))$ is a normal function. By the discussion above, a Bloch function is also a semi-Bloch function. Colonna [4] showed that a semi-Bloch function must be a normal function, and that there exist semi-Bloch functions which are not Bloch functions.

In this note, we give both an analytic characterization and a geometric characterization for semi-Bloch functions. In addition, we show by example that the sum of two semi-Bloch functions need not be a semi-Bloch function, and also that the square of a semi-Bloch function need not be a semi-Bloch function.

Received by the editors December 19, 1991 and, in revised form, April 2, 1992.

1991 Mathematics Subject Classification. Primary 30D45. 
For a function $f$ analytic on a region $Q$, we may consider $f$ as a composition $\pi \circ \tilde{f}$, where $\tilde{f}$ is a one-to-one mapping from $Q$ onto the Riemann surface $R_{f}$, and $\pi$ is the projection mapping from the covering surface $R_{f}$ onto $f(Q)$. Here, the local coordinate system on $R_{f}$ is taken so that, if $V$ is an open set in $R_{f}$, then the coordinates of $V$ are the same as the usual coordinates on $\pi(V)$. We say that a set $V$ on $R_{f}$ is a schlicht disk if there exists a disk $\Delta$ on the complex plane such that both $V$ is a component of $\pi^{-1}(\Delta)$ and the mapping $\pi: V \rightarrow \Delta$ is one-to-one. If $V$ is a schlicht disk, we will refer to the radius of $\Delta$ as the radius of $V$, and the center of $V$ will be the point which projects onto the center of $\Delta$. If $z \in Q$, we define

$$
\begin{aligned}
d_{f}(z)=\sup \{r & : V \text { is a schlicht disk on } R_{f} \\
& \text { with center } \tilde{f}(z) \text { and radius } r\} .
\end{aligned}
$$

If $Q=D$, it is known that if $V$ is a schlicht disk on $R_{f}$ with center $\tilde{f}\left(z_{0}\right)$ and radius $r$ then

$$
\left|f^{\prime}\left(z_{0}\right)\right|\left(1-\left|z_{0}\right|^{2}\right) \geq d_{f}\left(z_{0}\right)
$$

(see [2]). The main result of this paper is based on this inequality, and is the following theorem.

Theorem 1. Let $f$ be a function analytic in $D$. Then the following are equivalent:

(i) $f$ is a semi-Bloch function.

(ii) For each line $L$ in the complex plane,

$$
\sup \left\{\left|f^{\prime}(z)\right|\left(1-|z|^{2}\right): f(z) \in L\right\}=M_{L}<\infty .
$$

(iii) For each line $L$ in the complex plane,

$$
\sup \left\{d_{f}(z): f(z) \in L\right\}=D_{L}<\infty .
$$

Here, condition (ii) is an analytic criterion and condition (iii) is a geometric criterion for a function to be semi-Bloch.

\section{THE PROOF OF THEOREM 1}

We begin with the following simple lemma.

Lemma 1. If $f$ is a function analytic in $D$, and if $c$ is a constant, then $f$ is a semi-Bloch function if and only if $f+c$ is a semi-Bloch function.

Proof. For a fixed complex number $\lambda$,

$$
\exp \{\lambda(f(z)+c)\}=\exp (\lambda c) \exp \{\lambda f(z)\}=k \exp \{\lambda f(z)\},
$$

where $k=\exp (\lambda c)$ is a constant. It is easily verified that a constant multiple of a normal function is a normal function. Thus, if $f$ is a semi-Bloch function then $f+c$ is also a semi-Bloch function. The converse follows from the fact that $f=(f+c)+(-c)$.

Our second lemma is a kind of converse to a well-known theorem of Hurwitz. 
Lemma 2. Let $\Omega$ be a region bounded by a rectifiable Jordan curve, and let $\left\{F_{n}\right\}$ be a sequence of functions analytic on $\bar{\Omega}$, the closure of $\Omega$, such that the sequence $\left\{F_{n}\right\}$ converges uniformly to a function $F$ which is univalent on $\bar{\Omega}$. Let $K$ be a compact subset of $\Omega$, and let $E=F(K)$. There exists a positive integer $n_{0}$ such that, for each $n>n_{0}$, there exists a compact subset $K_{n}$ of $\Omega$ such that $F_{n}\left(K_{n}\right)=E$ and $F_{n}$ is univalent on $K_{n}$.

Proof. We note that $F$ is a homeomorphism from $\bar{\Omega}$ to $F(\bar{\Omega})$, and so $E$ is a compact subset of $F(\Omega)$. For each function $G$ analytic on $\bar{\Omega}$ and for each $w$ not in the set $G(\partial \Omega)$, define

$$
n(G, w)=\frac{1}{2 \pi i} \int_{\partial \Omega} \frac{G^{\prime}(z)}{G(z)-w} d z .
$$

Here, $n(G, w)$ is the number of times $G$ assumes the value $w$ in the region $\Omega$ (and, in fact, on the closed set $\bar{\Omega})$, and $n(G, w)$ must be a nonnegative integer. Since the set $F(\partial \Omega) \cap E=\varnothing$, and since the sequence $\left\{F_{n}\right\}$ converges uniformly to $F$ on $\bar{\Omega}$, there exists a positive integer $n_{1}$ such that $F_{n}(\partial \Omega) \cap E=\varnothing$ for each $n>n_{1}$. Further, if $w \in F(\Omega)-E$, then we have $n\left(F_{n}, w\right) \rightarrow n(F, w)=1$.

Suppose that there exists a sequence $\left\{w_{n_{k}}\right\}$ of points in $E$ such that $n\left(F_{n_{k}}, w_{n_{k}}\right) \neq 1$ for each $k$. Since $E$ is a compact set, by considering a subsequence, if necessary, we may assume that the subsequence $\left\{w_{n_{k}}\right\}$ converges to a point $w_{0} \in E$. But, because of the uniform convergence of the sequence $\left\{F_{n}\right\}$ on $\bar{\Omega}$, we have that

$$
\begin{aligned}
n\left(F_{n_{k}}, w_{n_{k}}\right) & =\frac{1}{2 \pi i} \int_{\partial \Omega} \frac{F_{n_{k}}^{\prime}(z)}{F_{n_{k}}(z)-w_{n_{k}}} d z \\
& \rightarrow \frac{1}{2 \pi i} \int_{\partial \Omega} \frac{F^{\prime}(z)}{F(z)-w_{0}} d z=1
\end{aligned}
$$

But this convergence requires that $n\left(F_{n_{k}}, w_{n_{k}}\right)=1$ for all but a finite number of $k$, contradicting our assumption that $n\left(F_{n_{k}}, w_{n_{k}}\right) \neq 1$ for each $k$. It follows that there exists $n_{0}$ such that $n\left(F_{n}, w\right)=1$ for each $n>n_{0}$ and each $w \in E$. To finish the proof, let $K_{n}=F_{n}^{-1}(E)$, and we have that $F_{n}: K_{n} \rightarrow E$ is oneto-one for each $n>n_{0}$. This proves the lemma.

We need one more lemma, which may be of independent interest.

Lemma 3. Let $f$ be a function analytic in $D$, let $L$ be a line in the complex plane, and define the two numbers $D_{L}$ and $M_{L}$ by

$$
D_{L}=\sup \left\{d_{f}(z): f(z) \in L\right\}
$$

and

$$
M_{L}=\sup \left\{\left|f^{\prime}(z)\right|\left(1-|z|^{2}\right): f(z) \in L\right\} .
$$

Then $D_{L}=\infty$ if and only if $M_{L}=\infty$.

Proof. It follows from the inequality $\left|f^{\prime}(z)\right|\left(1-|z|^{2}\right) \geq d_{f}(z)$ that $M_{L} \geq D_{L}$. Thus, to prove the lemma we need only show that $D_{L}=\infty$ whenever $M_{L}=\infty$.

We may assume, without loss of generality, that $L$ is the real line, since any line can be moved to the real line by a rotation and translation, and these motions will not change properties of the derivative or of schlicht disks. 
Suppose that $M_{L}=\infty$. Then there exists a sequence $\left\{z_{n}\right\}$ of points in $D$ such that $f\left(z_{n}\right) \in L$ and $\left|f^{\prime}\left(z_{n}\right)\right|\left(1-\left|z_{n}\right|^{2}\right) \rightarrow \infty$. Letting

$$
\gamma_{n}(z)=\left(z+z_{n}\right) /\left(1+\bar{z}_{n} z\right) \text { and } g_{n}(z)=f\left(\gamma_{n}(z)\right)-f\left(z_{n}\right),
$$

we have that $g_{n}(0)=0$ for each $n$ and $g_{n}^{\prime}(0) \rightarrow \infty$, so it follows that the sequence $\left\{g_{n}(z)\right\}$ is not a normal family in $D$. Thus, by a result of Zalcman [9, Lemma, p. 814], there exists a sequence of points $\left\{\zeta_{n}\right\}$ in $D$ and a sequence of positive real numbers $\rho_{n} \rightarrow 0$ such that, if $h_{n}(t)=g_{n}\left(\zeta_{n}+\rho_{n} t\right)$, then the sequence $\left\{h_{n}(t)\right\}$ converges uniformly on each compact subset of the complex plane to a nonconstant entire function $h(t)$. By a result of Ahlfors [1, p. 191] (or see [8, Theorem VI.8, p. 257]), for each positive integer $j$ sufficiently large, there exists a schlicht disk $V_{j}$ on $R_{h}$, the Riemann surface image of $h$, where $V_{j}$ lies over the disk $\{w:|w-j !|<j+1 / 10\}$. Let

$$
\Omega_{j}=\left\{t \in h^{-1}\left(V_{j}\right):|h(t)-j !| \leq j\right\}
$$

and let

$$
K_{j}=\left\{t \in \Omega_{j}:|h(t)-j !| \leq j / 2\right\} .
$$

Fixing $j$ large, we may apply Lemma 2 by letting $\Omega=\Omega_{j}, K=K_{j}, F=h$, and $F_{n}=h_{n}$ to obtain that there exists an integer $n_{0}$ (depending only on the fixed function $h$ and the fixed integer $j$ ) such that, for $n>n_{0}$ there exists a compact subset $S_{n}$ of $\Omega$ such that $h_{n}$ maps $S_{n}$ conformally onto $h(K)$, that is, the image of $h_{n}$ contains a schlicht disk with center over the real axis and radius $j / 2$. But the image of $h_{n}$ is a translation by the real number $-f\left(z_{n}\right)$ of the image of $f$, and thus it follows that $R_{f}$, the Riemann surface image of $f$, contains a schlicht disk with center on $L$ and radius $j / 2$. But since we may choose $j$ as large as we please, we conclude that $D_{L}=\infty$. This completes the proof of the lemma.

We now proceed to prove Theorem 1 .

Proof of Theorem 1. From Lemma 3, we have that (ii) and (iii) are equivalent. Thus, to prove the theorem, we need to show that (i) and (ii) are equivalent.

First, suppose $f$ is a semi-Bloch function, and let $L$ be a line in the complex plane. We may assume, without loss of generality, that $L$ is the imaginary axis, for if not, there exist complex numbers $\lambda$ and $\alpha$ with $|\lambda|=1$ such that $\{w=\lambda z+\alpha: z \in L\}$ is the imaginary axis, and, in view of Lemma 1, the function $\lambda f(z)+\alpha$ is a semi-Bloch function. Letting $g(z)=\exp (f(z))$ and $f(z) \in L$, we have $|g(z)|=1$ and thus

$$
\left(1-|z|^{2}\right) g^{\#}(z)=\frac{1}{2}\left(1-|z|^{2}\right)\left|f^{\prime}(z)\right| \leq c_{g},
$$

where $c_{g}=\sup \left\{g^{\#}(z)\left(1-|z|^{2}\right): z \in D\right\}<\infty$ since $g$ is a normal function. It follows that

$$
\sup \left\{\left(1-|z|^{2}\right)\left|f^{\prime}(z)\right|: f(z) \in L\right\}=M_{L} \leq 2 c_{g}<\infty .
$$

This proves that (i) implies (ii).

Now suppose that (ii) is satisfied. Let $\lambda \neq 0$ be given, let $L$ be a line such that $\{w=\lambda z: z \in L\}$ is the imaginary axis, and let $g_{\lambda}(z)=\exp \{\lambda f(z)\}$. Then, for $f(z) \in L$, we have that $\left|g_{\lambda}(z)\right|=1$, and

$$
\left(1-|z|^{2}\right) g_{\lambda}^{\#}(z)=\frac{1}{2}\left(1-|z|^{2}\right)|\lambda|\left|f^{\prime}(z)\right| \leq \frac{1}{2}|\lambda| M_{L} .
$$


Since $L$ contains an infinity of points, it follows from the five point theorem of the second author [5, Theorem 2, p. 493] that $g_{\lambda}$ is a normal function. This shows that $f$ is a semi-Bloch function and thus that (ii) implies (i). This completes the proof of the theorem.

\section{AN EXAMPle}

We now consider the question raised by Colonna [4], as to whether semiBloch functions are closed under addition. We answer this question in the negative.

Theorem 2. There exists a pair of semi-Bloch functions whose sum is not a semiBloch function. Also, there exists a semi-Bloch function whose square is not a semi-Bloch function.

Proof. Both examples will make use of the following construction. For $n \geq 3$, let $z_{n}=n !+i n$, and let $D_{n}$ be the circle with center at $z_{n}$ and radius $n-1$. For each $n$, let $L_{n}$ be the line segment from the origin to the point $z_{n}$, and let $S_{n}$ be a narrow channel (a connected open set) containing the portion of $L_{n}$ lying outside the unit disk such that the channels $S_{n}$ are mutually disjoint and $S_{n} \cap D_{j}=\varnothing$ for $n \neq j, n \geq 3, j \geq 3$. Let $\Omega$ be the union of the sets $S_{n}, D_{n}$, and the unit disk. Then $\Omega$ is a simply connected open set. Let $\phi$ denote the one-to-one conformal mapping from the unit disk $D$ onto $\Omega$. We claim that both functions $\phi(z)$ and $(\phi(z))^{2}-4$ are semi-Bloch functions.

If $L$ is any line in the complex plane, then it is easy to verify that the distance from each point of $L \cap \Omega$ to the boundary of $\Omega$ is bounded by a finite constant $C_{L}$ which depends only on the line. Letting $D_{L}=C_{L}$, we have, by Theorem 1 , that $\phi$ is a semi-Bloch function. If we consider the function $s(z)=z^{2}$, we note that each set $s\left(D_{n}\right)$ lies in a rectangle

$$
\begin{aligned}
\left\{(x, y):(n !-n+1)^{2}-\right. & (2 n-1)^{2} \leq x \leq(n !+n-1)^{2}-1, \\
& 2(n !-n+1) \leq y \leq 2(n !+n-1)(2 n-1)\},
\end{aligned}
$$

and it is easily verified that no line meets more than a finite number of these rectangles. In addition, the set $s\left(L_{n}\right)$ is a line from the origin to the point $\left((n !)^{2}-n^{2}, 2 n \cdot n !\right)$, this line has slope of the order of $2 /(n-1) !$, and $s\left(S_{n}\right)$ is a narrow channel containing most of $s\left(L_{n}\right)$. It is easily verified that the intersection of each line with the union of the sets $s\left(S_{n}\right)$ is bounded. Now set $h(z)=s \circ \phi(z)=(\phi(z))^{2}$. The reasoning above shows that, for each fixed line $L$, we have that $D_{L}=\sup \left\{d_{h}(z): h(z) \in L\right\}$ is finite, and it follows from Theorem 1 that $h$ is a semi-Bloch function. Then, from Lemma 1 , we have that $h(z)-4=(\phi(z))^{2}-4$ is also a semi-Bloch function.

We claim that $g(z)=(\phi(z)-2 i)^{2}=\left((\phi(z))^{2}-4\right)-4 i \phi(z)$ is not a semiBloch function. To see this, we will show that the function $S(\zeta)=(\zeta-2 i)^{2}$ sends the set $D_{n}, n \geq 3$, into a set which contains a disk centered on the real line and having radius at least $2 n !-(n-1)^{2}$. It is clear that the function $S$ is univalent on $D_{n}$, for each $n \geq 3$.

If $w_{0}$ is the point on the boundary of $D_{n}$ for which $\left|S\left(w_{0}\right)-S\left(z_{n}\right)\right|=$ $\inf \left\{\left|S(w)-S\left(z_{n}\right)\right|: w \in \partial D_{n}\right\}$, then

$$
\begin{gathered}
\left|\left(w_{0}-2 i\right)^{2}-\left(z_{n}-2 i\right)^{2}\right|=\left|w_{0}+z_{n}-4 i\right|\left|w_{0}-z_{n}\right|, \\
\left|w_{0}+z_{n}-4 i\right| \geq \operatorname{Re}\left(w_{0}+z_{n}\right) \geq 2 n !-(n-1), \quad \text { and }\left|w_{0}-z_{n}\right|=n-1 .
\end{gathered}
$$


However, the imaginary part of $\left(z_{n}-2 i\right)^{2}$ is $2(n-2)(n !)$ so the set $S\left(D_{n}\right)=$ $\left\{\omega=(\zeta-2 i)^{2}: \zeta \in D_{n}\right\}$ contains a schlicht disk centered at $S\left(z_{n}\right)$ with radius

$$
\left|\left(w_{0}-2 i\right)^{2}-\left(z_{n}-2 i\right)^{2}\right| \geq(2 n !-(n-1))(n-1),
$$

and thus $S\left(D_{n}\right)$ contains a schlicht disk centered on the real line with radius

$$
\left|\left(w_{0}-2 i\right)^{2}-\left(z_{n}-2 i\right)^{2}\right|-\operatorname{Im}\left(z_{n}-2 i\right)^{2} \geq 2 n !-(n-1)^{2} .
$$

Now it follows from Theorem 1 that $g(z)=(\phi(z)-2 i)^{2}$ is not a semi-Bloch function.

This same example proves both parts of Theorem 2, since $g$ is both a sum of two semi-Bloch functions, $\phi^{2}(z)-4$ and $-4 i \phi(z)$, and the square of the semi-Bloch function $\phi(z)-2 i$.

\section{REFERENCES}

1. L. V. Ahlfors, Zur Theorie der Überlagerungsflächen, Acta Math. 65 (1935), 157-194.

2. J. M. Anderson, J. Clunie, and Ch. Pommerenke, On Bloch functions and normal functions, J. Reine Angew. Math. 270 (1974), 12-37.

3. S. Axler, The Bergman space, the Bloch space, and commutators of multiplication operators, Duke Math. J. 53 (1986), 315-332.

4. F. Colonna, Bloch and normal functions and their relation, Rend. Circ. Mat. Palermo (2) 38 (1989), 161-180.

5. P. Lappan, A criterion for a meromorphic function to be normal, Comment. Math. Helv. 49 (1974), 492-495.

6. O. Lehto and K. I. Virtanen, Boundary behaviour and normal meromorphic functions, Acta Math. 97 (1957), 47-65.

7. K. F. Tse, On the sums and products of normal functions, Comment Math. Univ. St. Paul. 17 (1969), 63-72.

8. M. Tsuji, Potential theory in modern function theory, Maruzen, Tokyo, 1959.

9. L. Zalcman, A heuristic principle in complex function theory, Amer. Math. Monthly 82 (1975), 813-817.

Department of Mathematics, University of Joensue, SF-80101 Joensuu 10, Finland

E-mail address: AULASKARI@FINUJO.BITNET

Department of Mathematics, Michigan State University, East Lansing, Michigan 48824

E-mail address: 21144PALQMSU.BITNET 